TRANSACTIONS OF THE

AMERICAN MATHEMATICAL SOCIETY

Volume 355, Number 4, Pages 1443-1465

S 0002-9947(02)03220-8

Article electronically published on December 4, 2002

\title{
SQUARE-INTEGRABILITY MODULO A SUBGROUP
}

\author{
G. CASSINELLI AND E. DE VITO
}

\begin{abstract}
We prove a weak form of the Frobenius reciprocity theorem for locally compact groups. As a consequence, we propose a definition of squareintegrable representation modulo a subgroup that clarifies the relations between coherent states, wavelet transforms and covariant localisation observables. A self-contained proof of the imprimitivity theorem for covariant positive operator-valued measures is given.
\end{abstract}

\section{INTRODUCTION}

Given a locally compact group $G$, a closed subgroup $H$ and a unitary representation $\sigma$ of $H$, we prove the existence of an isomorphism between the commuting ring of the representation of $G$ unitarily induced by $\sigma$ and the space of admissible linear maps commuting with the action of $H$ (see Definition 1 below). This result can be viewed as a weak form of the Frobenius reciprocity theorem for locally compact groups (compare with [21]). In particular, it allows one to characterise the subrepresentations of the induced representation in terms of a condition of squareintegrability on the quotient space $G / H$. This equivalence suggests a natural definition of square-integrable representation modulo a subgroup that unifies many notions used in the literature in the context both of coherent states and of wavelet analysis (for a review of these topics, see [2]). Finally, we show that, given a unitary representation $U$ of $G$, there exists a positive operator-valued (POV) measure based on $G / H$ and covariant with respect to $U$ if and only if $U$ is square-integrable modulo $H$ (compare with [17] and 31]).

This latter result is a consequence of a generalisation of the classical Mackey imprimitivity theorem ([20], 4] ) to covariant POV measures based on $G / H$. We give a self-contained proof of this theorem for non-normalised POV measures, using as a key technical tool the notion of Gårding domain 24]. Some independent proofs were available for the case of normalised POV. Up to our knowledge, Poulsen [26] first proved it for Lie groups using elliptic regularity theorem. Other proofs, for locally compact groups, were given by Davies 9], Neumann [23], Scutaru 31, Cattaneo [7], and Castrigiano and Henrichs [8]. All these proofs reduce the problem to the projective case, either by the Neumark dilation theorem [28], or by Stinespring's theory of positive functions on $C^{*}$-algebras 32 .

Our proof is independent of both the Neumark theorem and the Mackey theorem, which are corollaries of our result. It is inspired by the proof of the Mackey imprimitivity theorem given by Orsted [24], as suggested by a remark in [8] (compare

Received by the editors November 15, 2001 and, in revised form, October 11, 2002.

2000 Mathematics Subject Classification. Primary 43A32, 43A85, 42C40.

Key words and phrases. Square-integrable representation, frame, localisation observable. 
also with [11, Ch. XXII, Sec. 3, Ex. 10]). In particular, we use a realisation of the induced representation outlined in an exercise of [11, Ch. XXII, Sec. 3, Ex. 10]. Our construction is a variation of the one given by Blattner [4], and, in our opinion, is very elementary and intrinsic. It does not use the notion of quasi-invariant measure, and the Hilbert space where the representation acts is a space of square-integrable functions (compare with Folland [13, Ch. 6]).

The paper is organised in the following way. In Sec. 2 we introduce the notation and we give our construction of the induced representation. In Sec. 3, we prove the weak Frobenius reciprocity theorem and we propose a definition of square-integrable representation modulo a subgroup. In Sec. 4 we prove the imprimitivity theorem for non-normalised transitive POV measures, and we characterise them in terms of square-integrable representations modulo a subgroup.

To avoid technical problems with integration theory, we assume that groups and Hilbert spaces are separable, but the results hold without this hypothesis.

\section{NotATiOn AND CONSTRUCTION OF THE INDUCED REPRESENTATION}

In this paper, $G$ is a locally compact second countable topological group and $H$ a closed subgroup of $G$. We denote by $\mu_{G}$ and $\mu_{H}$ left Haar measures on $G$ and $H$, respectively. Let $\Delta_{G}$ and $\Delta_{H}$ be the corresponding modular functions.

Let $X=G / H$ be the quotient space of the left cosets with the natural topology, and $p: G \rightarrow X$ the canonical projection, which is an open map. For all $g \in G$, we denote by $x \mapsto g[x]$ the action of $G$ on $X$. If $f$ is a function on $G$ and $g \in G$, we let $f^{g}$ be the map given by $\left(f^{g}\right)\left(g^{\prime}\right)=f\left(g^{-1} g^{\prime}\right)$, for all $g^{\prime} \in G$.

Given a locally compact second countable topological space $Y$, by a Radon measure on $Y$, we mean a positive measure defined on the $\sigma$-algebra $\mathcal{B}(Y)$ of Borel subsets of $Y$ that is finite on compact sets. Since the space is second countable, Radon measures are both outer and inner regular. In particular, Haar measures are Radon. We denote by $C_{c}(Y)$ the space of continuous complex functions on $Y$ with compact support, and by supp $f$ the support of a continuous function $f$.

We recall the following well-known result by Weil (for the proof see, for example, Prop. 2.48 of [13]).

Lemma 1. Let $f \in \mathrm{C}_{\mathrm{c}}(G)$, and let $K$ be its support. There is a unique $\tilde{f} \in \mathrm{C}_{\mathrm{c}}(X)$ such that, for all $g \in G$,

$$
\tilde{f}(p(g))=\int_{H} f(g h) d \mu_{H}(h)
$$

Moreover,

$$
\sup _{x \in X}|\tilde{f}(x)| \leq C_{K} \sup _{g \in G}|f(g)|,
$$

where $C_{K}$ is a constant depending only on the support of $f$. Finally, if $f^{\prime} \in \mathrm{C}_{\mathrm{c}}(X)$ [positive], there is $f \in \mathrm{C}_{\mathrm{c}}(G)$ [positive] such that $\tilde{f}=f^{\prime}$ and $p(\operatorname{supp} f)=\operatorname{supp} f^{\prime}$.

By Hilbert space, we mean a complex separable Hilbert space, with the scalar product $\langle\cdot, \cdot\rangle$, linear in the first variable, and $\|\cdot\|$ the corresponding norm. If $A$ is a (bounded) operator, we denote by $\|A\|$ the norm of $A$. By a representation of $G$, we mean a continuous (with respect to the strong operator topology) unitary 
representation of $G$ acting in a Hilbert space. Given a representation $\pi$ acting in $\mathcal{H}$, for all $f \in \mathrm{C}_{\mathrm{c}}(G)$, we define

$$
\pi(f)=\int_{G} f(g) \pi(g) d \mu_{G}(g)
$$

where the integral is in the strong operator topology. In particular, one has that, for all $g \in G$ and $f \in \mathrm{C}_{\mathrm{c}}(G)$,

$$
\pi(g) \pi(f)=\pi\left(f^{g}\right)
$$

We denote by $\mathcal{D}_{\pi}$ the Gårding domain of $\pi$, i.e.,

$$
\mathcal{D}_{\pi}=\operatorname{span}\left\{\pi(f) u \mid f \in \mathrm{C}_{\mathrm{c}}(G), u \in \mathcal{H}\right\} .
$$

One has the following properties.

Lemma 2. With the above notation, the Gärding domain of $\pi$ is a G-invariant dense subspace of $\mathcal{H}$. If $\pi^{\prime}$ is another representation of $G$ acting in $\mathcal{H}^{\prime}$ and $W$ is an operator from $\mathcal{H}$ to $\mathcal{H}^{\prime}$ intertwining $\pi$ and $\pi^{\prime}$, then

$$
W \mathcal{D}_{\pi} \subset \mathcal{D}_{\pi^{\prime}}
$$

Proof. Let $g \in G, f \in \mathrm{C}_{\mathrm{c}}(G)$ and $v \in \mathcal{H}$. By Eq.(2), $\pi(g) \pi(f) v=\pi\left(f^{g}\right) v$, and, since $f^{g} \in \mathrm{C}_{\mathrm{c}}(G)$, it follows that $\mathcal{D}_{\pi}$ is $G$-invariant. To show the density, given $v \in \mathcal{H}$ and $\epsilon>0$, since $\pi$ is continuous with respect to the strong operator topology, there is a compact neighbourhood $K$ of the identity such that, for all $g \in K,\|\pi(g) v-v\| \leq \epsilon$. Since $K$ contains a non-void open set, $\mu_{G}(K)>0$ and, by outer regularity of $\mu_{G}$, there is an open set $V \supset K$ with $\mu_{G}(V \backslash K) \leq \epsilon \mu_{G}(K)$. Let $f \in \mathrm{C}_{\mathrm{c}}(G)$ be such that $f(g)=1$ for all $g \in K, 0 \leq f(g) \leq 1$ for all $g \in G$, and $\operatorname{supp} f \subset V$. Then, defining $a=\frac{1}{\mu_{G}(K)}$,

$$
\begin{aligned}
\|\pi(a f) v-v\| & =\left\|a \int_{K}(\pi(g) v-v) d \mu_{G}(g)+a \int_{V \backslash K} f(g) \pi(g) v d \mu_{G}(g)\right\| \\
& \leq a \int_{K}\|\pi(g) v-v\| d \mu_{G}(g)+a \int_{V \backslash K} f(g)\|\pi(g) v\| d \mu_{G}(g) \\
& \leq \epsilon(1+\|v\|) .
\end{aligned}
$$

To show the second point, let $f \in \mathrm{C}_{\mathrm{c}}(G)$ and $v \in \mathcal{H}$; then

$$
\begin{aligned}
W \pi(f) v & =W \int_{G} f(g) \pi(g) v d \mu_{G}(g) \\
=\int_{G} f(g) W \pi(g) v d \mu_{G}(g) & =\int_{G} f(g) \pi^{\prime}(g) W v d \mu_{G}(g)=\pi^{\prime}(f) W v .
\end{aligned}
$$

If $G$ is a Lie group, the Gårding domain is usually defined by replacing $\mathrm{C}_{\mathrm{c}}(G)$ with $C_{c}^{\infty}(G)$ (see [33]). We adopt in this paper the definition of [24].

We now give a realisation of the induced representation, based on the following lemma.

Lemma 3. There is a continuous function $\theta: G \rightarrow[0,+\infty[$ such that, for all $g \in G$,

$$
\int_{H} \theta(g h) d \mu_{H}(h)=1
$$


and, for any compact subset $K$ of $G, K H \cap \operatorname{supp} \theta$ is compact.

Moreover, let $Y \in \mathcal{B}(G)$ be such that, for all $h \in H, \mu_{G}(Y h \backslash Y)=0$. Then $Y$ is negligible with respect to $\theta \mu_{G}$ if and only if it is negligible with respect to $\mu_{G}$, where $\theta \mu_{G}$ is the measure having density $\theta$ with respect to $\mu_{G}$.

Proof. The existence of $\theta$ is proven, for example, in Prop. 2 of [16]. With respect to the second part, if $\mu_{G}(Y)=0$, then $\int_{Y} \theta(g) d \mu_{G}(g)=0$. Conversely,

$$
\begin{aligned}
\int_{Y} d \mu_{G}(g) & =\int_{Y} \int_{H} \theta(g h) d \mu_{H}(h) d \mu_{G}(g) \\
& =\int_{H} \int_{Y} \theta(g h) d \mu_{G}(g) d \mu_{H}(h) \\
\left(g \mapsto g h^{-1}\right) & =\int_{H} \Delta_{G}\left(h^{-1}\right) \int_{Y h} \theta(g) d \mu_{G}(g) d \mu_{H}(h) \\
\left(\mu_{G}(Y h \backslash Y)=0\right) & =\int_{H} \Delta_{G}\left(h^{-1}\right) \int_{Y} \theta(g) d \mu_{G}(g) d \mu_{H}(h) \\
& =0 .
\end{aligned}
$$

Let $\sigma$ be a representation of $H$ acting in $\mathcal{K}$. Given $\theta$ as in Lemma 3, let $\mathcal{F}^{\sigma}$ be the subspace of functions $F$ from $G$ to $\mathcal{K}$ such that

- $F$ is $\mu_{G}$-measurable;

- given $h \in H$, for $\mu_{G}$-almost all $g \in G$,

$$
\sqrt{\frac{\Delta_{H}(h)}{\Delta_{G}(h)}} \sigma\left(h^{-1}\right) F(g)=F(g h) ;
$$

- $\int_{G}\|F(g)\|^{2} \theta(g) d \mu_{G}(g)<+\infty$.

We notice that, due to Lemma 3, a function $F$ satisfying Eq.(3) is $\mu_{G}$-measurable if and only if it is $\theta \mu_{G}$-measurable. So $F \in \mathcal{F}^{\sigma}$ if and only if $F \in L^{2}\left(G, \theta \mu_{G}, K\right)$ and Eq.(3) holds.

Given $v \in \mathcal{K}$ and $f \in \mathrm{C}_{\mathrm{c}}(G)$, let $F_{f, v}$ be the function from $G$ to $\mathcal{K}$ defined, for all $g \in G$, as

$$
\left(F_{f, v}\right)(g)=\int_{H} \sqrt{\frac{\Delta_{H}\left(h^{-1}\right)}{\Delta_{G}\left(h^{-1}\right)}} f(g h) \sigma(h) v d \mu_{H}(h) .
$$

Lemma 4. With the above notation, $\mathcal{F}^{\sigma}$ is a closed subspace of $L^{2}\left(G, \theta \mu_{G}, K\right)$ which does not depend on the choice of $\theta$, and each $F \in \mathcal{F}^{\sigma}$ is locally $\mu_{G}$-integrable. For each $f \in \mathrm{C}_{\mathrm{c}}(G)$ and $v \in \mathcal{K}, F_{f, v}$ is in $\mathcal{F}^{\sigma}$, it is continuous, and $\operatorname{supp} F_{f, v} \subset$ $(\operatorname{supp} f) H$. Finally, the space generated by the elements of the form $F_{f, v}$ is dense in $\mathcal{F}^{\sigma}$.

Proof. We claim that $\mathcal{F}^{\sigma}$ is closed. Indeed, let $\left(F_{n}\right)_{n \in \mathbb{N}}$ be a Cauchy sequence in $\mathcal{F}^{\sigma}$. Since $L^{2}\left(G, \theta \mu_{G}, K\right)$ is a Hilbert space, $\left(F_{n}\right)_{n \in \mathbb{N}}$ converges in $L^{2}$ and, possibly passing to a subsequence, $\theta \mu_{G}$-almost everywhere. Let $Y$ be the complement of the set of elements $g \in G$ such that $\left(F_{n}(g)\right)_{n \in \mathbb{N}}$ converges pointwise, and denote by $F(g)$ the limit. By hypothesis, $Y$ is $\theta \mu_{G}$-negligible, and, by unicity of the limit, $\left(F_{n}\right)_{n \in \mathbb{N}}$ converges to $F$ in $L^{2}$. Now let $h \in H$; by definition of $\mathcal{F}^{\sigma}$ and the fact 
that $\left(F_{n}\right)_{n}$ is denumerable, there exists $Y_{h} \in \mathcal{B}(G)$ such that $\mu_{G}\left(Y_{h}\right)=0$ and, for all $g \in G \backslash Y_{h}$ and $n \in \mathbb{N}$,

$$
\sqrt{\frac{\Delta_{H}(h)}{\Delta_{G}(h)}} \sigma\left(h^{-1}\right) F_{n}(g)=F_{n}(g h) .
$$

If $g \notin Y \cup Y_{h}$, passing to the limit, one has that $\left(F_{n}(g h)\right)_{n \in \mathbb{N}}$ converges and

$$
\sqrt{\frac{\Delta_{H}(h)}{\Delta_{G}(h)}} \sigma\left(h^{-1}\right) F(g)=F(g h) .
$$

In particular, $g h \notin Y$; that is, $Y h^{-1} \subset Y \cup Y_{h}$. Since $\mu_{G}\left(Y_{h}\right)=0$, it follows that $\mu_{G}\left(Y_{h} \backslash Y\right)=0$. By Lemma 3 it follows that $\mu_{G}(Y)=0$ and $\mu_{G}\left(Y \cup Y_{h}\right)=0$. So Eq.(4) holds $\mu_{G}$-almost everywhere; that is, $F \in \mathcal{F}^{\sigma}$.

We now prove that $\mathcal{F}^{\sigma}$ is independent of $\theta$. Let $\theta^{\prime}$ be a non-negative continuous function such that $\int_{H} \theta^{\prime}(g h) d \mu_{H}(h)=1$ for all $g \in G$. Let $F$ from $G$ to $K$ be $\mu_{G}$-measurable and such that Eq.(3) holds. Then

$$
\begin{aligned}
\int_{G}\|F(g)\|^{2} \theta(g) d \mu_{G}(g) & =\int_{G}\|F(g)\|^{2} \theta(g) \int_{H} \theta^{\prime}(g h) d \mu_{H}(h) d \mu_{G}(g) \\
& =\int_{H} \int_{G}\|F(g)\|^{2} \theta(g) \theta^{\prime}(g h) d \mu_{G}(g) d \mu_{H}(h) \\
\left(g \mapsto g h^{-1}\right) & =\int_{H} \int_{G}\left\|F\left(g h^{-1}\right)\right\|^{2} \theta\left(g h^{-1}\right) \theta^{\prime}(g) \Delta_{G}\left(h^{-1}\right) d \mu_{G}(g) d \mu_{H}(h) \\
\left(F \in \mathcal{F}^{\sigma}, h \mapsto h^{-1}\right) & =\int_{H} \int_{G}\|F(g)\|^{2} \theta(g h) \theta^{\prime}(g) d \mu_{G}(g) d \mu_{H}(h) \\
& =\int_{G}\|F(g)\|^{2} \theta^{\prime}(g) \int_{H} \theta(g h) d \mu_{H}(h) d \mu_{G}(g) \\
& =\int_{G}\|F(g)\|^{2} \theta^{\prime}(g) d \mu_{G}(g) .
\end{aligned}
$$

This shows the claim.

Now let $F \in \mathcal{F}^{\sigma}$; we prove that $F$ is locally $\mu_{G}$-integrable. Let $f \in \mathrm{C}_{\mathrm{c}}(G)$ be non-negative; then, as before,

$$
\begin{aligned}
\int_{G}\|F(g)\| f(g) d \mu_{G}(g) & =\int_{G}\|F(g)\| f(g) \int_{H} \theta(g h) d \mu_{H}(h) d \mu_{G}(g) \\
& =\int_{G}\|F(g)\| \theta(g) \int_{H} \sqrt{\frac{\Delta_{H}\left(h^{-1}\right)}{\Delta_{G}\left(h^{-1}\right)}} f(g h) d \mu_{H}(h) d \mu_{G}(g) \\
& =\int_{G}\|F(g)\| f^{\prime}(g) \theta(g) d \mu_{G}(g),
\end{aligned}
$$

where $f^{\prime}(g):=\int_{H} \sqrt{\frac{\Delta_{H}\left(h^{-1}\right)}{\Delta_{G}\left(h^{-1}\right)}} f(g h) d \mu_{H}(h)$ is continuous and supp $f^{\prime} \subset(\operatorname{supp} f) H$. By Lemma 3, $f^{\prime} \theta \in L^{2}\left(G, \mu_{G}\right)$, so that $\|F(g)\| f^{\prime}(g) \theta(g)$ is $\mu_{G}$-integrable, and hence $F$ is locally $\mu_{G}$-integrable.

The properties of $F_{f, v} \in \mathcal{F}^{\sigma}$ are clear (use the proof of Lemma 1). We show the density. Let $F \in \mathcal{F}^{\sigma}$ be such that, for all $f \in \mathrm{C}_{\mathrm{c}}(G)$ and $v \in \mathcal{K},\left\langle F, F_{f, v}\right\rangle=0$. Then, using the same argument as before and Tonelli's theorem, one can check that 
the map

$$
(g, h) \mapsto\langle F(g), \sigma(h) v\rangle \overline{f(g h)} \theta(g) \sqrt{\frac{\Delta_{H}\left(h^{-1}\right)}{\Delta_{G}\left(h^{-1}\right)}}
$$

is $\left(\mu_{G} \otimes \mu_{H}\right)$-integrable and

$$
\begin{aligned}
0 & =\left\langle F, F_{f, v}\right\rangle \\
& =\int_{G} \int_{H}\langle F(g), \sigma(h) v\rangle \overline{f(g h)} \theta(g) \sqrt{\frac{\Delta_{H}\left(h^{-1}\right)}{\Delta_{G}\left(h^{-1}\right)}} d \mu_{H}(h) d \mu_{G}(g) \\
\left.\left(g \mapsto g h^{-1}, h \mapsto h^{-1}\right)\right) & =\int_{G}\langle F(g), v\rangle \overline{f(g)} \int_{H} \theta(g h) d \mu_{H}(h) d \mu_{G}(g) \\
& =\int_{G}\langle F(g), v\rangle \overline{f(g)} d \mu_{G}(g) .
\end{aligned}
$$

By standard arguments, one has that $F(g)=0$ for $\mu_{G^{-}}$almost all $g \in G$; that is, $F=0$.

Define, for all $g \in G$ and $F \in \mathcal{F}^{\sigma}$,

$$
\left(L_{g}^{\sigma} F\right)\left(g^{\prime}\right)=F\left(g^{-1} g^{\prime}\right), \quad g^{\prime} \in G, \mu_{G} \text {-a.e. }
$$

One has the following result.

Proposition 1. Let $\sigma$ be a representation of $H$. Then $L^{\sigma}$ is a representation of $G$ acting in $\mathcal{F}^{\sigma}$ and is a realisation of the representation induced by $\sigma$ from $H$ to $G$. In particular, the Gairding domain of $L^{\sigma}$ is a subspace of continuous functions.

Proof. Given $g \in G$, we prove that $L_{g}^{\sigma}$ is a well-defined isometric operator in $\mathcal{F}^{\sigma}$. Let $F \in \mathcal{F}^{\sigma}$; then, for all $h \in H$ and for $\mu_{G^{-}}$almost all $g^{\prime} \in G$,

$$
\begin{aligned}
\left(L_{g}^{\sigma} F\right)\left(g^{\prime} h\right) & =F\left(g^{-1} g^{\prime} h\right) \\
& =\sqrt{\frac{\Delta_{H}(h)}{\Delta_{G}(h)}} \sigma\left(h^{-1}\right) F\left(g^{-1} g^{\prime}\right)=\sqrt{\frac{\Delta_{H}(h)}{\Delta_{G}(h)}} \sigma\left(h^{-1}\right)\left(L_{g}^{\sigma} F\right)\left(g^{\prime}\right) .
\end{aligned}
$$

Moreover,

$$
\begin{aligned}
\int_{G} \| & \left(g^{-1} g^{\prime}\right) \|^{2} \theta\left(g^{\prime}\right) d \mu_{G}\left(g^{\prime}\right) \\
& =\int_{G}\left\|F\left(g^{\prime}\right)\right\|^{2} \theta\left(g g^{\prime}\right) d \mu_{G}\left(g^{\prime}\right) \\
& =\int_{G}\left\|F\left(g^{\prime}\right)\right\|^{2} \theta\left(g g^{\prime}\right) \int_{H} \theta\left(g^{\prime} h\right) d \mu_{H}(h) d \mu_{G}\left(g^{\prime}\right) \\
& =\int_{H} \int_{G}\left\|F\left(g^{\prime}\right)\right\|^{2} \theta\left(g g^{\prime}\right) \theta\left(g^{\prime} h\right) d \mu_{G}\left(g^{\prime}\right) d \mu_{H}(h) \\
\left(g^{\prime} \mapsto g^{\prime} h^{-1}\right) & =\int_{H} \int_{G} \Delta_{G}\left(h^{-1}\right)\left\|F\left(g^{\prime}\right)\right\|^{2} \theta\left(g g^{\prime} h^{-1}\right) \theta\left(g^{\prime}\right) d \mu_{G}\left(g^{\prime}\right) d \mu_{H}(h) \\
\left(h \mapsto h^{-1}\right) & =\int_{G}\left\|F\left(g^{\prime}\right)\right\|^{2} \theta\left(g^{\prime}\right) \int_{H} \theta\left(g g^{\prime} h\right) d \mu_{H}(h) d \mu_{G}\left(g^{\prime}\right) \\
& =\int_{G}\left\|F\left(g^{\prime}\right)\right\|^{2} \theta\left(g^{\prime}\right) d \mu_{G}\left(g^{\prime}\right) .
\end{aligned}
$$

This proves that $L_{g}^{\sigma}$ is a well-defined isometric operator in $\mathcal{F}^{\sigma}$. 
In order to show that $g \mapsto L_{g}^{\sigma}$ is continuous, since $L_{g}^{\sigma}$ is isometric and by a density argument, it is enough to prove that, given $f \in \mathrm{C}_{\mathrm{c}}(G), v \in \mathcal{K}$ and $F^{\prime} \in \mathcal{F}^{\sigma}$, the map

$$
g \mapsto\left\langle L_{g}^{\sigma} F_{f, v}, F^{\prime}\right\rangle=\int_{G}\left\langle F_{f, v}\left(g^{-1} g^{\prime}\right), F^{\prime}(g)\right\rangle \theta(g) d \mu_{G}(g)
$$

is continuous. However, due to Lemma $4 F_{f, v}$ is continuous, and, due to Lemma 3) $\operatorname{supp} F_{f, v} \cap \operatorname{supp} \theta$ is compact, so that the thesis follows by the dominated convergence theorem.

We prove that $\mathcal{D}_{L^{\sigma}}$ is a subspace of continuous functions. Indeed, let $f \in \mathrm{C}_{\mathrm{c}}(G)$ and $F \in \mathcal{F}^{\sigma}$. Given $F^{\prime} \in \mathcal{F}^{\sigma}$, observe that the function on $G \times G$

$$
\Psi\left(g, g^{\prime}\right)=f(g)\left\langle F\left(g^{-1} g^{\prime}\right), F^{\prime}\left(g^{\prime}\right)\right\rangle \theta\left(g^{\prime}\right)
$$

is $\left(\mu_{G} \otimes \mu_{G}\right)$-measurable and

$$
\begin{aligned}
\int_{G} \int_{G} & \left|f(g)\left\langle F\left(g^{-1} g^{\prime}\right), F^{\prime}\left(g^{\prime}\right)\right\rangle \theta\left(g^{\prime}\right)\right| d \mu_{G}\left(g^{\prime}\right) d \mu_{G}(g) \\
\leq & \int_{G} \int_{G}|f(g)|\left\|F\left(g^{-1} g^{\prime}\right)\right\|\left\|F^{\prime}\left(g^{\prime}\right)\right\| \theta\left(g^{\prime}\right) d \mu_{G}\left(g^{\prime}\right) d \mu_{G}(g) \\
\leq & \int_{G}|f(g)|\left(\int_{G}\left\|F\left(g^{-1} g^{\prime}\right)\right\|^{2} \theta\left(g^{\prime}\right) d \mu_{G}\left(g^{\prime}\right)\right)^{\frac{1}{2}} \\
& \times\left(\int_{G}\left\|F^{\prime}\left(g^{\prime}\right)\right\|^{2} \theta\left(g^{\prime}\right) d \mu_{G}\left(g^{\prime}\right)\right)^{\frac{1}{2}} d \mu_{G}(g) \\
= & \int_{G}|f(g)|\left\|L_{g}^{\sigma} F\right\|_{\mathcal{F}^{\sigma}}\left\|F^{\prime}\right\|_{\mathcal{F}^{\sigma}} d \mu_{G}(g) \\
= & \int_{G}|f(g)|\|F\|_{\mathcal{F}^{\sigma}}\left\|F^{\prime}\right\|_{\mathcal{F}^{\sigma}} d \mu_{G}(g) .
\end{aligned}
$$

Since $f$ has compact support, the above integral is finite and, by Tonelli's theorem, $\Psi$ is integrable with respect to $\mu_{G} \otimes \mu_{G}$. Then

$$
\begin{aligned}
\left\langle L^{\sigma}(f) F, F^{\prime}\right\rangle & =\int_{G} f(g) \int_{G}\left\langle F\left(g^{-1} g^{\prime}\right), F^{\prime}\left(g^{\prime}\right)\right\rangle \theta\left(g^{\prime}\right) d \mu_{G}\left(g^{\prime}\right) d \mu_{G}(g) \\
& =\int_{G} \int_{G}\left\langle f(g) F\left(g^{-1} g^{\prime}\right), F^{\prime}\left(g^{\prime}\right)\right\rangle d \mu_{G}(g) \theta\left(g^{\prime}\right) d \mu_{G}\left(g^{\prime}\right) \\
\left(g \mapsto g^{\prime} g, g \mapsto g^{-1}\right) & =\int_{G} \int_{G}\left\langle\Delta_{G}\left(g^{-1}\right) f\left(g^{\prime} g^{-1}\right) F(g), F^{\prime}\left(g^{\prime}\right)\right\rangle d \mu_{G}(g) \theta\left(g^{\prime}\right) d \mu_{G}\left(g^{\prime}\right) \\
& =\int_{G}\left\langle(f \star F)\left(g^{\prime}\right), F^{\prime}\left(g^{\prime}\right)\right\rangle \theta\left(g^{\prime}\right) d \mu_{G}\left(g^{\prime}\right),
\end{aligned}
$$

where $(f \star F)\left(g^{\prime}\right)=\int_{G} \Delta_{G}\left(g^{-1}\right) f\left(g^{\prime} g^{-1}\right) F(g) d \mu_{G}(g)$, which is well defined since $F$ is locally $\mu_{G}$-integrable. Then, one has that $L^{\sigma}(f) F=f \star F$. The continuity of $f \star F$ is now a consequence of the dominated convergence theorem.

\section{A WEAK Form of the Frobenius RECIPRocity theOrem}

The following definition is a possible extension of the notion of admissible vector for square-integrable representations. We fix a function $\theta$ as given by Lemma 3 .

Definition 1. Let $\sigma$ be a representation of $H$ acting in $\mathcal{K}$ and $\pi$ a representation of $G$ acting in $\mathcal{H}$. A linear map $A: \mathcal{D}_{\pi} \rightarrow \mathcal{K}$ such that 
- for all $h \in H$ and $v \in \mathcal{D}_{\pi}$,

$$
\sigma(h) A v=\sqrt{\frac{\Delta_{H}(h)}{\Delta_{G}(h)}} A \pi(h) v ;
$$

- for all $v \in \mathcal{D}_{\pi}$, the map from $G$ to $\mathcal{K}$

$$
g \mapsto A \pi\left(g^{-1}\right) v:=\left(W_{A} v\right)(g)
$$

is $\mu_{G}$-measurable and

$$
\int_{G}\left\|A \pi\left(g^{-1}\right) v\right\|^{2} \theta(g) d \mu_{G}(g) \leq \beta\|v\|^{2},
$$

where $\beta$ is a positive constant independent of $v$, is called an admissible map for $\pi$ modulo $(H, \sigma)$.

The admissible maps modulo $(H, \sigma)$ give a characterisation of the bounded operators intertwining $\pi$ and the representation $L^{\sigma}$ induced by $\sigma$ (compare with Moore [21]).

Theorem 1. Let $\sigma$ be a representation of $H$ acting in $\mathcal{K}$ and $\pi$ a representation of $G$ acting in $\mathcal{H}$. Let $A: \mathcal{D}_{\pi} \rightarrow \mathcal{K}$ be an admissible map for $\pi$ modulo $(H, \sigma)$. Then:

- for all $v \in \mathcal{D}_{\pi}, W_{A} v \in \mathcal{D}_{L^{\sigma}} \subset \mathcal{F}^{\sigma}$ (in particular, $W_{A} v$ is a continuous function); and

- the linear map $v \mapsto W_{A} v$ extends to a unique bounded operator $W_{A}$, called the wavelet transform, from $\mathcal{H}$ to $\mathcal{F}^{\sigma}$ that intertwines $\pi$ and $L^{\sigma}$.

Conversely, given a bounded operator $W: \mathcal{H} \rightarrow \mathcal{F}^{\sigma}$ intertwining $\pi$ with $L^{\sigma}$, there is a unique admissible map $A$ (for $\pi$ modulo $(H, \sigma)$ ) such that, for all $v \in \mathcal{D}_{\pi}$, $W v=W_{A} v$.

Proof. Let $A$ be an admissible map. Given $v \in \mathcal{D}_{\pi}$ and $h \in H$, we have

$$
\begin{aligned}
\left(W_{A} v\right)(g h) & =A \pi\left(h^{-1} g^{-1}\right) v \\
=\sqrt{\frac{\Delta_{H}(h)}{\Delta_{G}(h)}} \sigma\left(h^{-1}\right) A \pi\left(g^{-1}\right) v & =\sqrt{\frac{\Delta_{H}(h)}{\Delta_{G}(h)}} \sigma\left(h^{-1}\right)\left(W_{A} v\right)(g),
\end{aligned}
$$

for all $g \in G$. Due to Eq.(6), one has that $W_{A} v \in \mathcal{F}^{\sigma}$ and $\left\|W_{A} v\right\| \leq \sqrt{\beta}\|v\|$. Since $\mathcal{D}_{\pi}$ is dense in $\mathcal{H}, v \mapsto W_{A} v$ extends to a unique bounded operator $W_{A}$. Moreover, if $g^{\prime} \in G$, then

$$
\left(W_{A} v\right)\left(g^{\prime-1} g\right)=A \pi\left(g^{-1} g^{\prime}\right) v=\left(W_{A} \pi\left(g^{\prime}\right) v\right)(g)
$$

for all $g \in G$, so that $W_{A}$ intertwines $\pi$ and $L^{\sigma}$. In particular, due to Lemma 2 $W_{A} \mathcal{D}_{\pi} \subset \mathcal{D}_{L^{\sigma}}$, and the elements of $\mathcal{D}_{L^{\sigma}}$ are continuous functions by Prop.1.

Conversely, let $W$ be a bounded operator from $\mathcal{H}$ to $L^{\sigma}$ intertwining $\pi$ and $\mathcal{F}^{\sigma}$. By Lemma 2 and Prop. 1, for all $v \in \mathcal{D}_{\pi}, W v$ is a continuous function, and we can define $A$ from $\mathcal{D}_{\pi}$ to $\mathcal{K}$ as $A v=(W v)(e)$, where $e$ is the identity of $G$. Given $h \in H$ and $v \in \mathcal{D}_{\pi}$,

$$
A \pi_{h} v=\left(W \pi_{h} v\right)(e)=\left(L_{h}^{\sigma} W v\right)(e)=(W v)\left(h^{-1}\right)=\sqrt{\frac{\Delta_{H}\left(h^{-1}\right)}{\Delta_{G}\left(h^{-1}\right)}} \sigma(h)(W v)(e),
$$

so that Eq.(5) holds. Moreover, if $g \in G$, then

$$
A \pi_{g^{-1}} v=\left(W \pi_{g^{-1}} v\right)(e)=\left(L_{g^{-1}}^{\sigma} W v\right)(e)=(W v)(g),
$$


so that $W_{A}=W$ on $\mathcal{D}_{\pi}$ and Eq.(6) is satisfied with $\beta=\|W\|^{2}$. Let $B$ be another admissible map such that, for all $v \in \mathcal{D}_{\pi}$,

$$
B \pi_{g^{-1}} v=(W v)(g), \quad g \in G \theta \mu_{G^{-}} \text {-a.e.. }
$$

Since both sides satisfy Eq. (3), by Lemma 3 the equality holds $\mu_{G}$-almost everywhere and, by continuity, everywhere. With the choice $g=e$, one has $B v=$ $(W v)(e)=A v$.

We add some comments. If a linear map $A$ satisfies Eq.(5) and is closable, its closure is semi-invariant with weight $h \mapsto \sqrt{\frac{\Delta_{H}(h)}{\Delta_{G}(h)}}$, in the sense of [12], and the measurability of $W_{A} v$ follows from the continuity of $\pi$. However, as shown by Example 1 below, there are admissible maps that are not closable. If $A$ is closable and $\pi$ is irreducible, condition (6) is equivalent to the existence of $v \in \mathcal{D}_{\pi}$ such that

$$
0<\int_{G}\left\|A \pi\left(g^{-1}\right) v\right\|^{2} \theta(g) d \mu_{G}(g)<+\infty
$$

(use the first part of the proof of Th. 3 of [12]).

If $X$ admits an invariant measure (in particular, if both $G$ and $H$ are unimodular), then Eq. (5) is the requirement that $A$ is an (algebraic) intertwining map between $\pi_{\mid H}$ and $\sigma$.

Assume now that $G$ is compact and $\pi$ irreducible. Since $\mathcal{H}$ is finite dimensional, it follows that $\mathcal{D}_{\pi}=\mathcal{H}$, and, taking into account that both $G$ and $H$ are unimodular, Eq.(5) is the condition that $A$ is an intertwining operator between $\pi_{\mid H}$ and $\sigma$. Finally, since the measure $\mu_{G}$ is bounded, Eq.(6) is trivially satisfied. Then, the space of admissible maps is precisely the set of intertwining operators between $\pi_{\mid H}$ and $\sigma$ and Theorem 1 reduces to the Frobenius reciprocity theorem for compact groups (see, for example, 13]).

In case $G$ is not compact, the following example shows that it is restrictive to assume that admissible maps are closable.

Example 1. In Theorem 1, let $\pi=L^{\sigma}$ and choose $W=I$. A simple computation shows that the admissible map $A$ such that $W_{A}=I$ is, for all $F \in \mathcal{D}_{L^{\sigma}}$,

$$
A F=F(e),
$$

which is clearly not closable (if $G$ is not discrete).

In particular, let $G$ be the Poincaré group $\mathbb{R}^{4} \times^{\prime} S O(3,1), H=\mathbb{R}^{4} \times^{\prime} S O(3)$ and $\sigma$ the one-dimensional representation of $H$ given by

$$
\sigma\left(\left(x_{0}, \vec{x}\right), R\right)=e^{i x_{0}}, \quad\left(x_{0}, \vec{x}\right) \in \mathbb{R}^{4}, R \in S O(3) .
$$

It is well known that $G / H$ has an invariant measure and $L^{\sigma}$ is irreducible, so that the multiples of the identity are the only intertwining operators. Due to the previous observation, in this example there are neither bounded nor closable admissible maps.

In general, Gårding domains do not have a natural topology such that Eq.(6) is equivalent to the continuity of the admissible maps. Hence, our result is not good enough to give a useful characterisation of the set of intertwining operators between $\pi$ and $L^{\sigma}$ (compare with so-called "intertwining number theorems"; see, for an exposition, 33], and the results contained in [27] for Lie groups). However, Theorem 1 allows us to characterise completely the representations that are equivalent to a subrepresentation of the induced one. 
Corollary 1. With the notation of Theorem [1, the following conditions are equivalent:

- the representation $\pi$ is equivalent to a subrepresentation of $L^{\sigma}$;

- there is an admissible map $A_{0}$ such that, for all $v \in \mathcal{D}_{\pi}$,

$$
\int_{G}\left\|A_{0} \pi\left(g^{-1}\right) v\right\|^{2} \theta(g) d \mu_{G}(g)=\|v\|^{2} ;
$$

- there is an admissible map $A$ such that, for all $v \in \mathcal{D}_{\pi}$,

$$
\alpha\|v\|^{2} \leq \int_{G}\left\|A \pi\left(g^{-1}\right) v\right\|^{2} \theta(g) d \mu_{G}(g) \leq \beta\|v\|^{2},
$$

where $0<\alpha \leq \beta$.

If any of the above three conditions is satisfied, we say that $\pi$ is square-integrable modulo $(H, \sigma)$.

Proof. Assume the first condition. Then there is an isometry $W$ intertwining $\pi$ and $L^{\sigma}$. Applying Theorem 1 to $W$, we see that there exists an admissible map $A$ such that $W=W_{A}$; and, since $W$ is isometric, Eq.(7) holds. Clearly Eq.(7) implies Eq.(8). Assume now the third condition. Then the corresponding wavelet operator $W_{A}$ satisfies, for all $v \in \mathcal{H}$,

$$
\sqrt{\alpha}\|v\| \leq\left\|W_{A} v\right\| \leq \sqrt{\beta}\|v\| .
$$

In particular, $W_{A}$ is injective, so that, by polar decomposition, there is an isometry $W_{0}$ such that $W_{A}=W_{0}\left|W_{A}\right|$. Since $W_{A}$ commutes with the action of $G, W_{0}$ intertwines $\pi$ and $L^{\sigma}$.

In the framework of wavelet analysis, Eq.(8) says that $\left\{A \pi\left(g^{-1}\right)\right\}_{g \in G}$ is an (operator-valued) frame in $\mathcal{H}$, and Eq. (7) says that this frame is tight. So one can restate Corollary 1 in the following way. A representation $\pi$ is square-integrable modulo $H$ if and only if the set $\left\{A \pi\left(g^{-1}\right)\right\}_{g \in G}$ is a frame for some admissible map $A$, and $A$ can always be chosen in such a way that the corresponding frame is tight.

Example 2. Assume that $\pi$ is irreducible, and let $H=\{e\}$, with $\sigma$ the trivial representation. Then, $\pi$ is square-integrable modulo $(H, \sigma)$ if and only if $\pi$ is square-integrable in the sense of Godement (see, for example, [16], if $G$ is unimodular, and [12], if $G$ is nonunimodular). In particular, there always exist bounded admissible maps $A=\langle\cdot, \eta\rangle$, where $\eta$ is in the domain of the formal degree of $\pi$ [12], such that Eq.(7) holds (compare with Example 1 above and Example 3 below).

If the admissible map is bounded, we have some more precise results.

Corollary 2. With the notation of Theorem 1 , let $A: \mathcal{H} \rightarrow \mathcal{K}$ be a bounded operator satisfying Eq.(5). Then

(1) the space $X$ has an invariant measure, i.e., $\frac{\Delta_{H}(h)}{\Delta_{G}(h)}=1$ for all $h \in H$;

(2) if A satisfies Eq.(6), the corresponding wavelet operator $W_{A}$ is given by

$$
\left(W_{A} v\right)(g)=A \pi\left(g^{-1}\right) v
$$

for all $g \in G$ and $v \in \mathcal{H}$;

(3) if $A$ satisfies Eq.(8), then $\pi$ is square-integrable modulo both $(H, \sigma)$ and $\left(H, \pi_{\mid H}\right)$. 
Proof. With respect to the first claim, it is clear that, if $A$ satisfies Eq.(55) for all $v \in \mathcal{D}_{\pi}$, then Eq. (5) holds for all $v \in \mathcal{H}$, i.e., $A$ is semi-invariant with weight $h \mapsto \sqrt{\frac{\Delta_{H}(h)}{\Delta_{G}(h)}}$ in the sense of [12]. However, $A$ is bounded, and this is possible only if $\frac{\Delta_{H}(h)}{\Delta_{G}(h)}=1$ for all $h \in H$ (compare with [12, Eq. 2]).

In order to show the second statement, let $v$ in $\mathcal{H}$ and $\left(v_{n}\right)_{n \in \mathbb{N}}$ in $\mathcal{D}_{\pi}$ be such that $v=\lim v_{n}$. Since $\left(\left(W_{A} v_{n}\right)(g)\right)_{n}$ converges pointwise to the continuous function $\psi_{v}(g)=A \pi\left(g^{-1}\right) v$, by Eq.(6) and Fatou's lemma, it follows that $\psi_{v} \in \mathcal{F}^{\sigma}$. On the other hand, $W_{A} v=\lim W_{A} v_{n}$ and, by unicity of the limit, $\psi_{v}=W_{A} v$.

Finally assume that Eq.(8) holds and let $A=U|A|$ be the polar decomposition of $A$. Clearly $|A|$ commutes with $\pi_{\mid H}$, and, taking into account that $U$ restricted to the range of $|A|$ is an isometry, Eq.(8) becomes

$$
\alpha\|v\|^{2} \leq \int_{G}\left\||A| \pi\left(g^{-1}\right) v\right\|^{2} \theta(g) d \mu_{G}(g) \leq \beta\|v\|^{2} .
$$

So, by Corollary 1, $\pi$ is square-integrable modulo $\left(H, \pi_{\mid H}\right)$.

We add a comment. Assume that $\pi$ admits a bounded admissible map $A$ such that the corresponding wavelet operator $W_{A}$ is injective (notice that this condition is weaker than Eq.(8)). By polar decomposition of $W_{A}$, it follows that $\pi$ is equivalent to a subrepresentation of $L^{\sigma}$, so that, according to Corollary $1 \pi$ is squareintegrable modulo $(H, \sigma)$ and there is an admissible map $A_{0}$ such that Eq.(7) holds. However, the following example, adapted from 14 and 15 , shows that $A_{0}$ can be unbounded (compare with the notions of weak and strong square-integrability in [14]).

Example 3. Let $G=\mathbb{R}$, and let $\pi$ be the representation acting on $\mathcal{H}=L^{2}\left(\mathbb{R}, \frac{d p}{\sqrt{2 \pi}}\right)$ as

$$
\left(\pi_{x} \phi\right)(p)=e^{-i x p} \phi(p), \quad p \in \mathbb{R} .
$$

Let $H=\{e\}$, and let $\sigma$ be the trivial representation acting on $\mathbb{C}$; then $L^{\sigma}$ is the regular representation of $\mathbb{R}$. The bounded maps from $\mathcal{H}$ to $\mathbb{C}$ are of the form $\langle\cdot, \eta\rangle$ for some vector $\eta \in \mathcal{H}$. Moreover, Condition ([6) is equivalent to the fact that the function

$$
x \mapsto A \pi(-x) \phi=\int_{\mathbb{R}} e^{i p x} \phi(p) \bar{\eta}(p) \frac{d p}{\sqrt{2 \pi}}=\mathcal{F}^{*}(\phi \bar{\eta})(x)
$$

is square-integrable, where $\mathcal{F}$ is the Fourier-Plancherel operator. For example, the above condition is satified if $\eta$ is a square-integrable continuous function going to zero at infinity. Hence, there exist bounded admissible maps for $\pi$, and the corresponding wavelet operators $W_{A}$ are given by $\mathcal{F}^{*} M_{\bar{\eta}}$, where $M_{\bar{\eta}}$ is the operator of multiplication by $\bar{\eta}$. If $\eta$ is strictly positive, $W_{A}$ is injective, so that $\pi$ is squareintegrable modulo $(H, \sigma)$. However, the range of $W_{A}$ is not closed for any choice of $\eta, \eta \neq 0$, so that $W_{A}$ cannot be an isometry. Hence, there are no bounded admissible maps $A_{0}$ such that Eq.(7) holds. One can check that the functions in $\mathcal{D}_{\pi}$ are of the form $\phi=f \psi$, where $\psi \in L^{2}$ and $f$ is a rapidly decreasing function. Moreover, reasoning as above, one has that the admissible maps $A_{0}$ such that $W_{A_{0}}$ is an isometry are of the form

$$
A_{0} \phi=\int_{\mathbb{R}} \phi(p) e^{i \alpha(p)} \frac{d p}{\sqrt{2 \pi}},
$$


where $\alpha$ is a real measurable function, and the integral is well defined since $\phi$ is in $\mathcal{D}_{\pi}$.

Our definition of square-integrability modulo a subgroup unifies many notions used in the literature for wavelet analysis and for generalised coherent states. For example:

(1) Square-integrability modulo the centre: $\pi$ is irreducible, $H$ is a central subgroup of $G, \sigma$ is the character of $H$ defined by the restriction of $\pi$ to $H$, and $A=\langle\cdot, v\rangle$, for some non-zero vector $v \in \mathcal{H}[5]$.

(2) Gilmore-Perelemov coherent states and $\alpha$-admissible vectors: $\pi$ is cyclic, $H$ is the stability subgroup, up to a phase factor, of some non-zero vector $v \in \mathcal{H}$ with respect to the action of $\pi_{\mid H}, \sigma$ is the corresponding character of $H$, and $A=\langle\cdot, v\rangle$ ([2], [25], [30] and references therein).

(3) Systems of coherent states: $\pi$ is arbitrary, $\sigma=\pi_{\mid H}$, and $A^{*} A$ is of trace class ([19], [31]).

(4) Vector coherent states and $V$-admissible vectors: $\pi$ is arbitrary, $\sigma$ is a finite dimensional representation contained in the restriction of $\pi$ to $H$, and $A$ is the projection onto the closed subspace left invariant by $\sigma$ ([1], [2], [29] and references therein).

(5) Weak and strong integrability: $\pi$ is arbitrary, $H=\{e\}$ with the trivial representation, and $A=\langle\cdot, v\rangle$ for some $v \in \mathcal{H}$ ([3], 14] and [15]).

\section{THE IMPRIMITIVITY THEOREM FOR SYSTEMS OF COVARIANCE}

We start with the definition of covariant localisation observable.

Definition 2. Given a representation $\pi$ of $G$ acting in $\mathcal{H}$, a map $E$ from the Borel subsets $\mathcal{B}(X)$ of $X$ to the set of positive operators in $\mathcal{H}$ such that

(1) $E(\emptyset)=0$;

(2) $E(X)$ is injective;

(3) for any disjoint sequence $\left(Y_{n}\right)_{n \in \mathbb{N}}$ in $\mathcal{B}(X)$,

$$
E\left(\bigcup_{n} Y_{n}\right)=\sum_{n} E\left(Y_{n}\right)
$$

where the series converges in the strong operator topology; and

(4) for all $g \in G$ and $Y \in \mathcal{B}(X)$,

$$
\pi_{g} E(Y) \pi_{g}^{-1}=E(g[Y]),
$$

is called a localisation observable based on $X$, covariant with respect to $\pi$ and acting in $\mathcal{H}$. Moreover,

- if $E(X)=I, E$ is said to be normalised, and

- if, for all $Y \in \mathcal{B}(X), E(Y)$ is a projection operator, $E$ is said to be projective.

The first and third requirements are the fact that $E$ is a POV measure on $X$, and the fourth is that $(\pi, E)$ is a system of $G$-covariance [7] or a generalised imprimitivity [17] (see, also, [9], [26]). The second requirement is not a constraint, since the kernel of $E(X)$ is invariant with respect to the action of $\pi$ and is contained in the kernel of $E(Y)$ for any $Y \in \mathcal{B}(X)$. Finally, if $E$ is projective, then it is necessarily normalised and commutative, and $(\pi, E)$ is a system of imprimitivity, [20] (see, also, [13]). The reason to introduce the name covariant localisation observable, instead of system of $G$-covariance, is to stress the nonsymmetric roles played by the representation 
$\pi$ and the POV measure $E$ in Definition 2. In doing so, we adopt the terminology from Quantum Mechanics (see, for example, [6], [18]).

The notion of equivalence is the natural one. Indeed, if $E_{1}$ and $E_{2}$ are localisation observables covariant with respect to $\pi_{1}$ and $\pi_{2}$, respectively, they are equivalent if there is a unitary operator $T$ intertwining $\pi_{1}$ and $\pi_{2}$ such that

$$
E_{2}(Y) T=T E_{1}(Y) \quad \forall Y \in \mathcal{B}(X) .
$$

Example 4. Let $\sigma$ be a representation of $H$, and $L^{\sigma}$ the corresponding induced representation acting in $\mathcal{F}^{\sigma}$. For all $Y \in \mathcal{B}(X)$, let $E^{\sigma}(Y)$ be the operator in $\mathcal{F}^{\sigma}$ defined by

$$
\left(E^{\sigma}(Y) F\right)(g)=\chi_{Y}(p(g)) F(g), \quad g \in G, \mu_{G} \text {-a.e. },
$$

where $F \in \mathcal{F}^{\sigma}$ and $\chi_{Y}$ is the characteristic function of the subset $Y$. It is well known (see, for example, [13]) that $Y \mapsto E(Y)$ is a projective localisation observable based on $X$ and covariant with respect to $L^{\sigma}$.

Now let $T$ be a positive operator in $\mathcal{F}^{\sigma}$ commuting with $L^{\sigma}$. Define $\mathcal{F}_{T}^{\sigma}$ as the closure of the range of $T$, let $L_{T}^{\sigma}$ be the restriction of $L^{\sigma}$ to $\mathcal{F}_{T}^{\sigma}$, and, for any $Y \in \mathcal{B}(X)$, let $E^{\sigma}(Y)_{T}=T E^{\sigma}(Y) T$, regarded as an operator in $\mathcal{F}_{T}^{\sigma}$. Clearly, the map $Y \mapsto E^{\sigma}(Y)_{T}$ is localisation observable based on $X$ and covariant with respect to $L_{T}^{\sigma}$. The next theorem will show that, up to an equivalence, all the localisation observables are of this form.

Theorem 2. Let $\pi$ be a representation of $G$ acting in $\mathcal{H}$, and $E$ a localisation observable based on $X$ and covariant with respect to $\pi$. There is a unique (up to an equivalence class) representation $\sigma_{E}$ of $H$ and an isometry $W$ from $\mathcal{H}$ to $\mathcal{F}^{\sigma_{E}}$ such that

$$
\begin{aligned}
W \pi(g) & =L^{\sigma_{E}}(g) W, \quad g \in G, \\
E(Y) & =E(X)^{\frac{1}{2}} W^{*} E^{\sigma_{E}}(Y) W E(X)^{\frac{1}{2}}, \quad Y \in \mathcal{B}(X), \\
\mathcal{F}^{\sigma} & =\overline{\operatorname{span}}\left\{E^{\sigma_{E}}(Y) W v \mid Y \in \mathcal{B}(X), v \in \mathcal{H}\right\} .
\end{aligned}
$$

Moreover, $E$ is projective if and only if $W E(X)^{\frac{1}{2}}$ is unitary. Finally, if $E^{\prime}$ is another localisation observable equivalent to $E$, then $\sigma_{E^{\prime}}$ is equivalent to $\sigma_{E}$.

Proof. We split the proof into seven steps.

Step 1). We define an operator-valued linear form $M$ on $\mathrm{C}_{\mathrm{c}}(G)$ associated with the POV measure $E$.

Given $u \in \mathcal{H}$, let $d\langle E(x) u, u\rangle$ be the bounded (Radon) measure on $X$,

$$
Y \mapsto\langle E(Y) u, u\rangle,
$$

having total mass $\langle E(X) u, u\rangle \leq\|u\|^{2}\|E(X)\|$ and satisfying, due to Eq.(99),

$$
\int_{X} f(g[x]) d\langle E(x) u, u\rangle=\int_{X} f(x) d\left\langle E(x) \pi_{g} u, \pi_{g} u\right\rangle,
$$

for all $g \in G$.

Given $f \in \mathrm{C}_{\mathrm{c}}(G)$ and $u \in \mathcal{H}$, by Lemma 1, the integral

$$
\int_{X}\left(\int_{H} f(g h) d \mu_{H}(h)\right) d\langle E(p(g)) u, u\rangle
$$

is well defined, linear in $f$, quadratic in $u$, and bounded by

$$
C_{f} \sup _{g \in G}|f(g)|\|u\|^{2}\|E(X)\|,
$$


where $C_{f}$ is a constant depending only on the support on $f$. For fixed $f \in \mathrm{C}_{\mathrm{c}}(G)$, by the polarisation identity, there is a unique operator $M(f)$ on $\mathcal{H}$ such that, for all $u \in \mathcal{H}$,

$$
\begin{aligned}
\langle M(f) u, u\rangle & =\int_{X}\left(\int_{H} f(g h) d \mu_{H}(h)\right) d\langle E(p(g)) u, u\rangle, \\
\|M(f)\| & \leq 4 C_{f} \sup _{g \in G}|f(g)|\|E(X)\|
\end{aligned}
$$

(the factor 4 is due to the polarisation identity). By Eq.(13), it follows that, for all $g \in G$ and $f \in \mathrm{C}_{\mathrm{c}}(G)$,

$$
\pi(g) M(f) \pi(g)=M\left(f^{g}\right) .
$$

We claim that, for all $h \in H$ and $f \in \mathrm{C}_{\mathrm{c}}(G)$,

$$
M(f(\cdot h))=\Delta_{H}\left(h^{-1}\right) M(f),
$$

where $f(\cdot h)$ is the function $g \mapsto f(g h)$. Indeed, if $u \in \mathcal{H}$,

$$
\begin{aligned}
\langle M(f(\cdot h)) u, u\rangle & =\int_{X}\left(\int_{H} f(g s h) d \mu_{H}(s)\right) d\langle E(p(g)) u, u\rangle, \\
\left(s \mapsto s h^{-1}\right) & =\Delta_{H}\left(h^{-1}\right) \int_{X}\left(\int_{H} f(g s) d \mu_{H}(s)\right) d\langle E(p(g)) u, u\rangle \\
& =\Delta_{H}\left(h^{-1}\right)\langle M(f) u, u\rangle .
\end{aligned}
$$

Step 2). We show that, if $u, v \in \mathcal{D}_{\pi}$, there is a unique continuous function $\phi_{u, v}$ defined on $G$ such that

$$
\langle M(f) u, v\rangle=\int_{G} f(g) \phi_{u, v}(g) d \mu_{G}(g), \quad f \in \mathrm{C}_{\mathrm{c}}(G) .
$$

The unicity is clear, since $\mu_{G}$ is a Radon measure and $\phi_{u, v}$ is continuous. To prove the existence, given $u, v \in \mathcal{H}$, we define a linear form on $\mathrm{C}_{\mathrm{c}}(G \times G)$ in the following way. Let $\beta \in \mathrm{C}_{\mathrm{c}}(G \times G)$, and let $K \subset G \times G$ be its support, with $K_{1}$ and $K_{2}$ the projection of $K$ on the first and second space, respectively. For fixed $g \in G$, the map $g^{\prime} \mapsto \beta\left(g^{\prime}, g\right)=: \beta_{g}$ is in $\mathrm{C}_{\mathrm{c}}(G)$. So the function

$$
G \ni g \mapsto \psi(g):=\left\langle M\left(\beta_{g}\right) \pi(g) u, v\right\rangle \in \mathbb{C}
$$

is well defined. We claim that $\psi \in \mathrm{C}_{\mathrm{c}}(G)$ Indeed, given $g_{1}, g_{2} \in G$, we have

$$
\begin{aligned}
\left|\psi\left(g_{1}\right)-\psi\left(g_{2}\right)\right|= & \left|\left\langle M\left(\beta_{g_{1}}\right) \pi\left(g_{1}\right) u, v\right\rangle-\left\langle M\left(\beta_{g_{2}}\right) \pi\left(g_{2}\right) u, v\right\rangle\right| \\
\leq & \left|\left\langle M\left(\beta_{g_{1}}\right)\left(\pi\left(g_{1}\right)-\pi\left(g_{2}\right) u\right), v\right\rangle\right|+\left|\left\langle M\left(\beta_{g_{2}}-\beta_{g_{1}}\right) \pi\left(g_{2}\right) u, v\right\rangle\right| \\
(\text { Eq.(14) }) \leq & 4 C_{K_{1}}\|v\|\|E(X)\|\left(\sup _{G \times G}\left|\beta\left(g^{\prime}, g\right)\right|\left\|\pi\left(g_{1}\right)-\pi\left(g_{2}\right) u\right\|\right. \\
& \left.+\sup _{g^{\prime} \in G}\left|\beta\left(g^{\prime}, g_{1}\right)-\beta\left(g^{\prime}, g_{2}\right)\right|\|u\|\right),
\end{aligned}
$$

since $\pi$ and $\beta$ are continuous, also $\psi$ is continuous. By Eq.(14), one has that, for all $g \in G$,

$$
|\psi(g)| \leq 4 C_{K_{1}} \sup _{g^{\prime} \in G}\left|\beta\left(g^{\prime}, g\right)\right|\|u\|\|v\|\|E(X)\| .
$$

So $\operatorname{supp} \psi \subset K_{2}$ and $\psi \in \mathrm{C}_{\mathrm{c}}(G)$. 
It follows that there is an operator $\Lambda(\beta)$ in $\mathcal{H}$ such that

$$
\begin{aligned}
\langle\Lambda(\beta) u, v\rangle & =\int_{G} \psi(g) d \mu_{G}(g)=\int_{G}\left\langle M\left(\beta_{g}\right) \pi(g) u, v\right\rangle d \mu_{G}(g), \\
\|\Lambda(\beta)\| & \leq C_{K} \sup _{G \times G}\left|\beta\left(g^{\prime}, g\right)\right|\|E(X)\|,
\end{aligned}
$$

where $C_{K}=4 C_{K_{1}} \mu_{G}\left(K_{2}\right)$ depends only on the support of $\beta$. In particular, if $f_{1}, f_{2} \in \mathrm{C}_{\mathrm{c}}(G)$, one has

$$
\begin{aligned}
\left\langle\Lambda\left(f_{1} \otimes f_{2}\right) u, v\right\rangle & =\int_{G} f_{2}(g)\left\langle M\left(f_{1}\right) \pi(g) u, v\right\rangle d \mu_{G}(g) \\
& =\left\langle M\left(f_{1}\right) \pi\left(f_{2}\right) u, v\right\rangle,
\end{aligned}
$$

and, for all $h \in H$ and $\beta \in \mathrm{C}_{\mathrm{c}}(G \times G)$,

$$
\begin{aligned}
\langle\Lambda(\beta(\cdot h, \cdot)) u, v\rangle & =\int_{G}\left\langle M\left(\beta_{g}(\cdot h)\right) \pi(g) u, v\right\rangle d \mu_{G}(g) \\
(\text { Eq. 160) }) & =\Delta_{H}\left(h^{-1}\right) \int_{G}\left\langle M\left(\beta_{g}(\cdot)\right) \pi(g) u, v\right\rangle d \mu_{G}(g) \\
& =\Delta_{H}\left(h^{-1}\right)\langle\Lambda(\beta(\cdot, \cdot)) u, v\rangle .
\end{aligned}
$$

For fixed $u, v \in \mathcal{H}$, by Eq. (17), it follows that the linear form $\beta \mapsto\langle\Lambda(\beta) u, v\rangle$ is continuous with respect to the natural topology of $\mathrm{C}_{\mathrm{c}}(G \times G)$, and there are a measurable complex function $\eta_{u, v}$ of modulo 1 and a Radon measure $\lambda_{u, v}$ on $G \times G$ such that

$$
\langle\Lambda(\beta) u, v\rangle=\int_{G \times G} \beta\left(g, g^{\prime}\right) \eta_{u, v}\left(g, g^{\prime}\right) d \lambda_{u, v}\left(g, g^{\prime}\right) ;
$$

see, for example, [10, Ch. XIII, Sec. 16].

Given $f_{1}, f_{2} \in \mathrm{C}_{\mathrm{c}}(G)$ and $u, v \in \mathcal{H}$, then, for all $f \in \mathrm{C}_{\mathrm{c}}(G)$,

$$
\begin{aligned}
\left\langle M(f) \pi\left(f_{1}\right) u, \pi\left(f_{2}\right) v\right\rangle & \\
= & \int_{G} \overline{f_{2}(g)}\left\langle M(f) \pi\left(f_{1}\right) u, \pi(g) v\right\rangle d \mu_{G}(g) \\
\text { (Eq. (15) })= & \int_{G} \overline{f_{2}(g)}\left\langle M\left(f^{g^{-1}}\right) \pi\left(g^{-1}\right) \pi\left(f_{1}\right) u, v\right\rangle d \mu_{G}(g) \\
\text { (Eqs.(2) },(\underline{18})= & \int_{G} \overline{f_{2}(g)}\left\langle\Lambda\left(f^{g^{-1}} \otimes f_{1}^{g^{-1}}\right) u, v\right\rangle d \mu_{G}(g) \\
= & \int_{G} \overline{f_{2}(g)} \int_{G \times G} f\left(g g_{1}\right) f_{1}\left(g g_{2}\right) \eta_{u, v}\left(g_{1}, g_{2}\right) d \lambda_{u, v}\left(g_{1}, g_{2}\right) d \mu_{G}(g) \\
= & \int_{G \times G \times G} f\left(g g_{1}\right) f_{1}\left(g g_{2}\right) \overline{f_{2}(g)} \eta_{u, v}\left(g_{1}, g_{2}\right) d \mu_{G}(g) d \lambda_{u, v}\left(g_{1}, g_{2}\right) \\
\left(g \mapsto g g_{1}^{-1}\right)= & \int_{G \times G \times G} f(g) f_{1}\left(g g_{1}^{-1} g_{2}\right) \overline{f_{2}\left(g g_{1}^{-1}\right)} \\
& \times \Delta_{G}\left(g_{1}^{-1}\right) \eta_{u, v}\left(g_{1}, g_{2}\right) d \mu_{G}(g) d \lambda_{u, v}\left(g_{1}, g_{2}\right) \\
= & \int_{G} f(g) \phi_{\pi\left(f_{1}\right) u, \pi\left(f_{2}\right) v} d \mu_{G}(g),
\end{aligned}
$$

where, for all $g \in G$,

$$
\phi_{\pi\left(f_{1}\right) u, \pi\left(f_{2}\right) v}(g)=\int_{G \times G} f_{1}\left(g g_{1}^{-1} g_{2}\right) \overline{f_{2}\left(g g_{1}^{-1}\right)} \Delta_{G}\left(g_{1}^{-1}\right) \eta_{u, v}\left(g_{1}, g_{2}\right) d \lambda_{u, v}\left(g_{1}, g_{2}\right),
$$


which is a continuous function, since $f_{1}, f_{2} \in \mathrm{C}_{\mathrm{c}}(G)$. By linearity, it follows that, for all $u, v \in \mathcal{D}_{\pi}$ and $f \in \mathrm{C}_{\mathrm{c}}(G)$, there is a continuous function $\phi_{u, v}$ such that

$$
\langle M(f) u, v\rangle=\int_{G} f(g) \phi_{u, v} d \mu_{G}(g) .
$$

Step 3). We construct a Hilbert space $\mathcal{K}$, which will carry the representation $\sigma_{E}$.

Let $\phi$ be the sequilinear form defined on $\mathcal{D}_{\pi} \times \mathcal{D}_{\pi}$ as $\phi(u, v)=\phi_{u, v}(e)$. Clearly, if $f_{1}, f_{2} \in \mathrm{C}_{\mathrm{c}}(G)$ and $u, v \in \mathcal{H}$, then

$$
\begin{aligned}
\phi\left(\pi\left(f_{1}\right) u, \pi\left(f_{2}\right) v\right) & =\int_{G \times G} f_{1}\left(g_{1}^{-1} g_{2}\right) \overline{f_{2}\left(g_{1}^{-1}\right)} \Delta_{G}\left(g_{1}^{-1}\right) \eta_{u, v}\left(g_{1}, g_{2}\right) d \lambda_{u, v}\left(g_{1}, g_{2}\right) \\
& =\left\langle\Lambda\left(f_{1} \bullet f_{2}\right) u, v\right\rangle
\end{aligned}
$$

with $\left(f_{1} \bullet f_{2}\right)\left(g_{1}, g_{2}\right)=f_{1}\left(g_{1}^{-1} g_{2}\right) \overline{f_{2}\left(g_{1}^{-1}\right)} \Delta_{G}\left(g_{1}^{-1}\right)$. By Eqs.(21), (201) and the dominated convergence theorem, the map

$$
\left(g, g^{\prime}\right) \mapsto \phi\left(\pi(g) \pi\left(f_{1}\right) u, \pi\left(g^{\prime}\right) \pi\left(f_{2}\right) v\right)
$$

is continuous on $G \times G$ and

$$
\phi_{\pi\left(f_{1}\right) u, \pi\left(f_{2}\right) v}(g)=\phi\left(\pi\left(g^{-1}\right) \pi\left(f_{1}\right) u, \pi\left(g^{-1}\right) \pi\left(f_{2}\right) v\right) .
$$

By linearity, it follows that, for all $u, v \in \mathcal{D}_{\pi}$,

$$
\left(g, g^{\prime}\right) \mapsto \phi\left(\pi(g) u, \pi\left(g^{\prime}\right) v\right) \text { is continuous }
$$

and, for all $f \in \mathrm{C}_{\mathrm{c}}(G)$,

$$
\int_{G} f(g) \phi\left(\pi\left(g^{-1}\right) u, \pi\left(g^{-1}\right) v\right) d \mu_{G}(g)=\int_{G} f(g) \phi_{u, v}(g) d \mu_{G}(g)=\langle M(f) u, v\rangle .
$$

The form $\phi$ is non-negative, since, by construction,

$$
\begin{aligned}
\langle M(f) u, u\rangle & =\int_{G} f(g) \phi_{u, u}(g) d \mu_{G}(g) \\
& =\int_{X}\left(\int_{H} f(g h) d \mu_{H}(h)\right) d\langle E(p(g)) u, u\rangle,
\end{aligned}
$$

which is clearly non-negative; then $\phi_{u, u}(g) \geq 0 \mu_{G}$-almost everywhere and, since $\phi_{u, u}$ is continuous, $\phi_{u, u}(e) \geq 0$.

Let $\mathcal{K}$ be the closure of the quotient space of $\mathcal{D}_{\pi}$ over the kernel of $\phi$, with respect to the scalar product induced by $\phi$, and let $A$ be the map from $\mathcal{D}_{\pi}$ to $\mathcal{K}$ mapping $v \in \mathcal{D}_{\pi}$ into its equivalence class, viewed in a natural way as an element of $\mathcal{K}$.

We claim that $\mathcal{K}$ is separable (so that $\mathcal{K}$ is indeed a Hilbert space). Since $\mathcal{N}:=A \mathcal{D}_{\pi}$ is dense in $\mathcal{K}$, it is sufficient to show that $\mathcal{N}$ is separable. Since $G$ is second countable, there is a denumerable family $\left\{f_{n}\right\}_{n \in \mathbb{N}}$ in $\mathrm{C}_{\mathrm{c}}(G)$ such that for any $f \in \mathrm{C}_{\mathrm{c}}(G)$ and $\epsilon>0$ there is a compact set $K$ and $f_{n}$ satisfying

$$
\begin{aligned}
\operatorname{supp}\left(f-f_{n}\right) & \subset K, \\
\sup _{g \in G}\left|f(g)-f_{n}(g)\right| & <\epsilon .
\end{aligned}
$$

Moreover, since $\mathcal{H}$ is separable, there is a denumerable family $\left\{u_{m}\right\}_{m \in \mathbb{N}}$ dense in $\mathcal{H}$. We claim that $\left\{A \pi\left(f_{n}\right) u_{m}\right\}_{n, m \in \mathbb{N}}$ is dense in $\mathcal{N}$. 
Indeed, given $f \in \mathrm{C}_{\mathrm{c}}(G)$ and $u \in \mathcal{H}$, let $f_{n}$ and $u_{m}$ be such that Eq.(23) holds, $\left\|u-u_{m}\right\|<\epsilon$ and $\left\|u_{m}\right\| \leq 2\|u\|$. Then

$$
\begin{aligned}
\left\|A \pi(f) u-A \pi\left(f_{n}\right) u_{m}\right\|_{\mathcal{K}} & \leq\left\|A \pi\left(f-f_{n}\right) u_{m}\right\|_{\mathcal{K}}+\left\|A \pi(f)\left(u-u_{m}\right)\right\|_{\mathcal{K}} \\
& =\sqrt{\phi_{\pi(l) u_{m}, \pi(l) u_{m}}}+\sqrt{\phi_{\pi(f) v, \pi(f) v}},
\end{aligned}
$$

where $l=f-f_{n}$ and $v=u-u_{m}$. Then, using Eqs. (17) and (20),

$$
\begin{aligned}
\phi_{\pi(l) u_{m}, \pi(l) u_{m}} & =\left\langle\Lambda(l \bullet l) u_{m}, u_{m}\right\rangle \\
& \leq C_{K} \sup _{G \times G}\left|l\left(g_{1}^{-1} g_{2}\right) \overline{l\left(g_{1}^{-1}\right)} \Delta_{G}\left(g_{1}^{-1}\right)\right|\|E(X)\|\left\|u_{m}\right\|^{2} \\
& \leq C_{K} \sup _{g \in K}\left(\Delta_{G}\left(g^{-1}\right)\right)\left(\sup _{g \in G}\left|f(g)-f_{n}(g)\right|\right)^{2}\|E(X)\|\left\|u_{m}\right\|^{2} \\
& \leq 4 C_{K} \sup _{g \in K}\left(\Delta_{G}\left(g^{-1}\right)\right)\|E(X)\|\|u\|^{2} \epsilon^{2},
\end{aligned}
$$

where $C_{K}$ depends only on $K$. In the same way,

$$
\begin{aligned}
\phi_{\pi(f) v, \pi(f) v} & =\langle\Lambda(f \bullet f) v, v\rangle \\
& \leq C_{K} \sup _{G \times G}\left|f\left(g_{1}^{-1} g_{2}\right) \overline{f\left(g_{1}^{-1}\right)} \Delta_{G}\left(g_{1}^{-1}\right)\right|\|E(X)\|\left\|u-u_{m}\right\|^{2} \\
& \leq C_{K} \sup _{g \in K}\left(\Delta_{G}\left(g^{-1}\right)\right)\|E(X)\|\left(\sup _{g \in G}|f(g)|\right)^{2} \epsilon^{2} .
\end{aligned}
$$

From the above inequalities, one has that

$$
\left\|A \pi(f) u-A \pi\left(f_{n}\right) u_{m}\right\|_{\mathcal{K}} \leq C^{\prime} \epsilon,
$$

where $C^{\prime}$ is a suitable constant depending only on $f$ and $u$. Since the set $\{A \pi(f) u\}$ spans $A \mathcal{D}_{\pi}$, the claim follows.

Step 4). We define a representation $\sigma_{E}$, denoted in the following simply by $\sigma$, and an isometry $W$ satisfying Eqs. (101), (11) and (12).

To this aim, we first prove that, for all $h \in H$ and $u, v \in \mathcal{D}_{\pi}$,

$$
\phi(\pi(h) u, \phi(h) v)=\frac{\Delta_{H}\left(h^{-1}\right)}{\Delta_{G}\left(h^{-1}\right)} \phi(u, v) .
$$

We can always assume that $u=v=\pi(f) w$ for some $f \in \mathrm{C}_{\mathrm{c}}(G)$ and $w \in \mathcal{H}$. Then, by Eq.(2),

$$
\begin{aligned}
\phi(\pi(h) \pi(f) w, \pi(h) \pi(f) w) & =\phi\left(\pi\left(f^{h}\right) w, \pi\left(f^{h}\right) w\right) \\
& =\left\langle\Lambda\left(f^{h} \bullet f^{h}\right) w, w\right\rangle \\
& =\Delta_{G}(h)\langle\Lambda((f \bullet f)(\cdot h, \cdot)) w, w\rangle \\
\text { (Eq.(19) }) & =\frac{\Delta_{H}\left(h^{-1}\right)}{\Delta_{G}\left(h^{-1}\right)}\langle\Lambda((f \bullet f)(\cdot, \cdot)) w, w\rangle \\
& =\frac{\Delta_{H}\left(h^{-1}\right)}{\Delta_{G}\left(h^{-1}\right)} \phi(\pi(f) w, \pi(f) w) .
\end{aligned}
$$

From Eq.(24), it follows that there is an isometric operator $\sigma_{h}$ in $\mathcal{K}$ such that, for all $h \in H$,

$$
\sigma_{h} A u=\sqrt{\frac{\Delta_{H}(h)}{\Delta_{G}(h)}} A \pi_{h} u, \quad u \in \mathcal{D}_{\pi} .
$$


We claim that $h \mapsto \sigma$ is a representation of $H$. The algebraic properties are clear, and, since $\sigma_{h}$ is isometric for all $h \in H$, it is sufficient to show that, given $u, v \in \mathcal{D}_{\pi}$, the map

$$
h \mapsto\left\langle\sigma_{h} A u, A v\right\rangle_{\mathcal{K}}=\sqrt{\frac{\Delta_{H}(h)}{\Delta_{G}(h)}} \phi\left(\pi_{h} u, v\right)
$$

is continuous. This fact follows from Eq.(21). We denote by $\sigma$ the representation defined by Eq.(25).

Moreover, we claim that $A$ is an admissible map with respect to $(\sigma, H)$. By construction, Eq.(5) is satisfied. Moreover, for all $u, v \in \mathcal{D}_{\pi}$, the map $g \mapsto$ $\left\langle A \pi\left(g^{-1}\right) u, A v\right\rangle_{\mathcal{K}}$ is continuous by Eq.(21), and hence, $g \mapsto A \pi\left(g^{-1}\right) u$ is $\mu_{G^{-}}$ measurable. Now let $f \in \mathrm{C}_{\mathrm{c}}(G)$ be non-negative and $u \in \mathcal{D}_{\pi}$. By Eq.(22),

$$
\begin{aligned}
\langle M(f) u, u\rangle & =\int_{G} f(g) \phi\left(\pi\left(g^{-1}\right) u, \pi\left(g^{-1}\right) u\right) d \mu_{G}(g) \\
& =\int_{G} f(g)\left\|A \pi\left(g^{-1}\right) u\right\|^{2} d \mu_{G}(g) \\
& =\int_{G} f(g)\left\|A \pi\left(g^{-1}\right) u\right\|^{2} \int_{H} \theta(g h) d \mu_{H}(h) d \mu_{G}(g) \\
\left(g \mapsto g h^{-1}\right) & =\int_{H} \int_{G} \Delta_{G}\left(h^{-1}\right) f\left(g h^{-1}\right)\left\|A \pi\left(h g^{-1}\right) u\right\|^{2} \theta(g) d \mu_{G}(g) d \mu_{H}(h) \\
\left(\text { Eq.(55), } h \mapsto h^{-1}\right) & =\int_{G}\left(\int_{H} f(g h) d \mu_{H}(h)\right)\left\|A \pi\left(g^{-1}\right) u\right\|^{2} \theta(g) d \mu_{G}(g) .
\end{aligned}
$$

By the definition of $M(f)$ and with the notation of Lemma 1, one has that

$$
\int_{X} \tilde{f}(x) d\langle E(x) u, u\rangle=\int_{G} \tilde{f}(p(g))\left\|A \pi\left(g^{-1}\right) u\right\|^{2} \theta(g) d \mu_{G}(g) .
$$

By Lemma 1, there is a positive sequence $\left(f_{n}\right)_{n \in \mathbb{N}}$ in $\mathrm{C}_{\mathrm{c}}(G)$ such that $\left(\tilde{f}_{n}\right)_{n}$ is a partition of unit on $X$. Then

$$
\begin{aligned}
\langle E(X) u, u\rangle & =\sum_{n} \int_{X} \tilde{f}_{n}(x) d\langle E(x) u, u\rangle \\
(\mathrm{Eq} \cdot(26)) & =\sum_{n} \int_{G} \tilde{f}_{n}(p(g))\left\|A \pi\left(g^{-1}\right) u\right\|^{2} \theta(g) d \mu_{G}(g) .
\end{aligned}
$$

By the monotone convergence theorem, the map $g \mapsto\left\|A \pi\left(g^{-1}\right) u\right\|^{2} \theta(g)$ is $\mu_{G^{-}}$ integrable and

$$
\langle E(X) u, u\rangle=\int_{G}\left\|A \pi\left(g^{-1}\right) u\right\|^{2} \theta(g) d \mu_{G}(g) .
$$

This shows that $A$ is an admissible map.

Let $W_{A}$ be the corresponding wavelet operator. From (27), one has that, for all $u \in \mathcal{D}_{\pi},\left\langle W_{A} u, W_{A} u\right\rangle=\langle E(X) u, u\rangle$; that is, by density, $W_{A}^{*} W_{A}=E(X)$. By Theorem 11, $W_{A}$ intertwines $\pi$ with $L^{\sigma}$, and, by the definition of localisation observable, $E(X)$ is injective. Then, by polar decomposition, there is an isometry $W$ such that $W_{A}=W E(X)^{\frac{1}{2}}$. Since $W$ intertwines $\pi$ with $L^{\sigma}, W$ satisfies Eq.(10). 
To prove Eq.(11), let $f \in \mathrm{C}_{\mathrm{c}}(G)$ and $u \in \mathcal{D}_{\pi}$. Then, using the definition of $E^{\sigma}$,

$$
\begin{aligned}
\int_{X} \tilde{f}(x) d\left\langle W_{A}^{*} E^{\sigma}(x) W_{A} u, u\right\rangle & =\int_{G} \tilde{f}(p(g))\left\langle\left(W_{A} u\right)(g),\left(W_{A} u\right)(g)\right\rangle_{\mathcal{K}} \theta(g) d \mu_{G}(g) \\
& =\int_{G} \tilde{f}(p(g))\left\|A \pi\left(g^{-1}\right) u\right\|^{2} \theta(g) d \mu_{G}(g) \\
\text { (Eq. (26) }) & =\int_{X} \tilde{f}(x) d\langle E(x) u, u\rangle .
\end{aligned}
$$

By the Riesz-Markov theorem and the surjectivity of the map $f \mapsto \tilde{f}$, see Lemma 1 it follows that, for all $Y \in \mathcal{B}(X)$ and $u \in \mathcal{D}_{\pi}$,

$$
\langle E(Y) u, u\rangle=\left\langle W_{A}^{*} E^{\sigma}(Y) W_{A} u, u\right\rangle .
$$

By density and the definition of $W$, the claim follows.

For Eq.(12), it is sufficient to prove that the closed subspace

$$
\mathcal{M}:=\left\{F \in \mathcal{F}^{\sigma} \mid\left\langle F, E^{\sigma}(Y) W_{A} v\right\rangle=0, \forall Y \in \mathcal{B}(X), v \in \mathcal{H}\right\}
$$

is the null space. From Eq.(9) and the fact that $W_{A}$ commutes with the action of $G$, it follows that $\mathcal{M}$ is a $G$-invariant closed subspace of $\mathcal{F}^{\sigma}$. In particular, if $f \in \mathrm{C}_{\mathrm{c}}(G)$ and $F^{\prime} \in \mathcal{M}$, then $F=L^{\sigma}(f) F^{\prime}$ is in $\mathcal{M}$ and, due to Proposition 1, $F$ is a continuous function. Now let $Y \in \mathcal{B}(X)$ and $v \in \mathcal{D}_{\pi}$; then

$$
\begin{aligned}
0 & =\left\langle F, E^{\sigma}(Y) W_{A} v\right\rangle \\
& =\int_{p^{-1}(Y)}\left\langle F(g),\left(W_{A} v\right)(g)\right\rangle_{\mathcal{K}} \theta(g) d \mu_{G}(g) .
\end{aligned}
$$

Since $v \in \mathcal{D}_{\pi}$, by Theorem $\square$ we see that $W_{A} v$ is continuous, and, hence, also the map $\left\langle F(g),\left(W_{A} v\right)(g)\right\rangle_{\mathcal{K}}$ is continuous. Due to this and the fact that $F, W_{A} u \in \mathcal{F}^{\sigma}$, one has that for all $h \in H$ and for all $g \in G$,

$$
\left\langle F(g h),\left(W_{A} v\right)(g h)\right\rangle_{\mathcal{K}}=\frac{\Delta_{H}(h)}{\Delta_{G}(h)}\left\langle F(g),\left(W_{A} v\right)(g)\right\rangle_{\mathcal{K}} .
$$

Let

$$
Y^{\prime}=\left\{g \in G \mid\left\langle F(g),\left(W_{A} v\right)(g)\right\rangle_{\mathcal{K}} \leq 0\right\},
$$

which is closed. Since $\frac{\Delta_{H}(h)}{\Delta_{G}(h)}$ is strictly positive, due to Eq. (29), for all $h \in H$ we have $Y^{\prime} h=Y^{\prime}$. Defining $Y=p\left(Y^{\prime}\right)$, which is closed since $p$ is an open map, one has that $Y^{\prime}=p^{-1}(Y)$, and, using Eq.(28), it follows that $\left\langle F(g),\left(W_{A} v\right)(g)\right\rangle_{\mathcal{K}}=$ 0 for $\theta \mu_{G^{-}}$-almost all $g \in Y^{\prime}$. By Lemma 3 ( $\left(Y^{\prime} h=Y^{\prime}\right)$ and the continuity, $\left\langle F(g),\left(W_{A} v\right)(g)\right\rangle_{\mathcal{K}}=0$ for all $g \in Y^{\prime}$. Repeating the above argument, one concludes that $\left\langle F(g),\left(W_{A} v\right)(g)\right\rangle_{\mathcal{K}}=0$ for all $g \in G$. By the definition of $W_{A}$ we have $\left\langle F(g), A \pi\left(g^{-1}\right) v\right\rangle_{\mathcal{K}}=0$, and, since $A \mathcal{D}_{\pi}$ is dense in $\mathcal{K}$ and $\pi\left(g^{-1}\right)$ is unitary, it follows that $F(g)=0$ for all $g \in G$; that is, $F=0$. Since $\left\{\pi(f) F^{\prime} \mid f \in \mathrm{C}_{\mathrm{c}}(G)\right.$, $\left.F^{\prime} \in \mathcal{M}\right\}$ is dense in $\mathcal{M}$, one has that $\mathcal{M}=0$. The claim is now clear.

Step 5). We show that $\sigma$ is unique up to an equivalence.

Let $\tau$ be another representation of $H$ acting in $\mathcal{K}^{\prime}$, and $W^{\prime}$ the isometry from $\mathcal{H}$ to $L^{\tau}$ such that Eqs.(10), (11) and (12) hold. By Theorem11 there is an admissible map $B$ from $\pi$ with respect to $(H, \tau)$ such that $W_{B}=W E(X)^{\frac{1}{2}}$. Given $f \in \mathrm{C}_{\mathrm{c}}(G)$ 
and $u \in \mathcal{D}_{\pi}$, applying Eq.(11) with $W^{\prime}$ instead of $W$, one has that

$$
\begin{aligned}
\langle M(f) u, u\rangle & =\int_{X} \tilde{f}(x) d\left\langle E^{\tau}(x) W_{B} u, W_{B} u\right\rangle \\
& =\int_{G} \tilde{f}(p(g))\left\|B \pi\left(g^{-1}\right) u\right\|^{2} \theta(g) d \mu_{G}(g) \\
& =\int_{H} \int_{G} f(g h)\left\|B \pi\left(g^{-1}\right) u\right\|^{2} \theta(g) d \mu_{G}(g) d \mu_{H}(h) \\
\left(g \mapsto g h^{-1}, h \mapsto h^{-1}\right) & =\int_{H} \int_{G} f(g)\left\|B \pi\left(g^{-1}\right) u\right\|^{2} \theta(g h) d \mu_{G}(g) d \mu_{H}(h) \\
& =\int_{G} f(g)\left\|B \pi\left(g^{-1}\right) u\right\|^{2} d \mu_{G}(g) .
\end{aligned}
$$

On the other hand, due to Eq.(22),

$$
\int_{G} f(g)\left\|A \pi\left(g^{-1}\right) u\right\|^{2} d \mu_{G}(g)=\int_{G} f(g)\left\|B \pi\left(g^{-1}\right) u\right\|^{2} d \mu_{G}(g) .
$$

By standard arguments, it follows that $\|A u\|_{\mathcal{K}}=\|B u\|_{\mathcal{K}^{\prime}}$. Hence, there is a unique isometric operator $t$ from $\mathcal{K}$ to $\mathcal{K}^{\prime}$ such that

$$
t A u=B u, \quad u \in \mathcal{D}_{\pi} .
$$

By the definition of $\sigma$ and the fact that $B$ satisfies Eq.(5), $t$ intertwines $\sigma$ and $\tau$. Since Eq.(12) holds for both $W$ and $W^{\prime}$, one has that $t$ is indeed unitary. This shows that $\tau$ is equivalent to $\sigma$.

Step 6$)$. We characterise the condition that $E$ is projective.

Assume now that $W E(X)^{\frac{1}{2}}$ is unitary; then, since $E^{\sigma}$ is projective, see Example 4 and Eq.(11), it follows that also $E$ is projective. Conversely, assume that $E$ is projective. Since $E$ is normalised, $E(X)^{\frac{1}{2}}=I$ and $W=W_{A}$. For all $Y, Z \in \mathcal{B}(X)$ and $u, v \in \mathcal{H}$,

$$
\begin{aligned}
\left\langle W W^{*} E^{\sigma}(Y) W u, E^{\sigma}(Z) W v\right\rangle & =\left\langle W E(Y) u, E^{\sigma}(Z) W v\right\rangle \\
& =\left\langle W^{*} E^{\sigma}(Z) W E(Y) u, v\right\rangle \\
& =\langle E(Z \cap Y) u, v\rangle \\
& =\left\langle W^{*} E^{\sigma}(Z \cap Y) W E(Y) u, v\right\rangle \\
& =\left\langle E^{\sigma}(Y) W u, E^{\sigma}(Z) W v\right\rangle,
\end{aligned}
$$

since both $E$ and $E^{\sigma}$ are projective. By Eq. (12), $\left\{E^{\sigma}(Y) W u\right\}$ is total in $\mathcal{F}^{\sigma}$, so that $W W^{*}=I$, and, since $W$ is an isometry, $W$ is unitary.

Step 7). We show that the equivalence class of $E$ defines the equivalence class of $\sigma_{E}$.

Let $E^{\prime}$ be as in the statement of the theorem, and let $T$ be a unitary operator such that

$$
\begin{aligned}
E(Y)^{\prime} T & =T E(Y), \quad Y \in \mathcal{B}(X), \\
\pi(g)^{\prime} T & =T \pi(g), \quad g \in G,
\end{aligned}
$$

where, here and in the following, we denote by a prime the objects that refer to $E^{\prime}$. Given $f \in \mathrm{C}_{\mathrm{c}}(G)$, from Eq.(3I) it follows that $M^{\prime}(f) T=T M(f)$. Now let $u \in \mathcal{D}_{\pi}$ and $v \in \mathcal{D}_{\pi^{\prime}}$; then by Lemma 2 Tu $T u \mathcal{D}_{\pi^{\prime}}$, and, by Eq.(22) applied first to $E^{\prime}$ and 
then to $E$,

$$
\begin{aligned}
\int_{G} f & (g) \phi^{\prime}\left(\pi^{\prime}\left(g^{-1}\right) T u, \pi^{\prime}\left(g^{-1}\right) v\right) d \mu_{G}(g) \\
& =\left\langle M(f)^{\prime} T u, v\right\rangle \\
& =\left\langle M(f) u, T^{*} v\right\rangle \\
& =\int_{G} f(g) \phi\left(\pi\left(g^{-1}\right) u, \pi\left(g^{-1}\right) T^{*} v\right) d \mu_{G}(g) .
\end{aligned}
$$

By standard arguments, one has that $\phi^{\prime}(T u, v)=\phi\left(u, T^{*} v\right)$. Since $T$ is unitary, for all $u, v \in \mathcal{D}_{\pi}$,

$$
\phi^{\prime}(T u, T v)=\phi(u, v) .
$$

It follows that there is a unitary operator $t$ from $\mathcal{K}$ to $\mathcal{K}^{\prime}$ such that, for all $u \in \mathcal{D}_{\pi}$,

$$
t A u=A^{\prime} t u \text {. }
$$

By the definition of $\sigma$ and $\sigma^{\prime}$ and Eq.(31), one has that, for all $h \in H$ and $u \in \mathcal{D}_{\pi}$,

$$
\sigma^{\prime}(h) t u=t \sigma(h) u \text {. }
$$

Hence, by density, $\sigma^{\prime}$ is equivalent to $\sigma$.

We add some comments. With the notation of Example 4 and $\sigma=\sigma_{E}$, equation (11) implies that $E$ is equivalent to a localisation observable of the form $E_{T}^{\sigma}$, where $T=W E(X)^{\frac{1}{2}} W^{*}$ is a positive operator on $\mathcal{F}^{\sigma}$. In particular, there always exists a normalised localisation observable $E_{0}$, covariant with respect to $\pi$ and acting in $\mathcal{H}$, such that

$$
E(Y)=E(X)^{\frac{1}{2}} E_{0}(Y) E(X)^{\frac{1}{2}}, \quad Y \in \mathcal{B}(X) .
$$

If $E$ is normalised, Eq. (12) implies that $\left(\mathcal{F}^{\sigma}, E^{\sigma}\right)$ is the dilation of $(\mathcal{H}, E)$ in the sense of the Neumark dilatation theorem. Moreover, since our proof is independent of the Mackey imprimitivity theorem, it contains the Mackey result as a particular case and one can easily show that two projective localisation observables $E$ and $E^{\prime}$ are equivalent if and only if $\sigma_{E}$ and $\sigma_{E^{\prime}}$ are equivalent. However, if $E$ and $E^{\prime}$ are not projective, one has only the only if part, as shown by the following example.

Example 5. Let $G=\mathcal{T}$ be the one-dimensional torus, and let $H=\{e\}$. Denote by $\left(f_{1}, f_{2}\right)$ the canonical basis of $\mathcal{H}:=\mathbb{C}^{2}$, and define, for all $z \in \mathcal{T}$ and $Y \in \mathcal{B}(\mathcal{T})$,

$$
\begin{aligned}
\pi(z) & =z\left\langle\cdot, f_{1}\right\rangle f_{1}+z^{2}\left\langle\cdot, f_{2}\right\rangle f_{2}, \\
E(Y) & =\mu(Y)\left\langle\cdot, f_{1}\right\rangle f_{1}+\mu(Y)\left\langle\cdot, f_{2}\right\rangle f_{2}, \\
E^{\prime}(Y) & =E(Y)+\int_{Y} \bar{z} d \mu(z)\left\langle\cdot, f_{1}\right\rangle f_{2}+\int_{Y} z d \mu(z)\left\langle\cdot, f_{2}\right\rangle f_{1},
\end{aligned}
$$

where $\mu$ is the normalised Haar measure on $\mathcal{T}$. By direct computation, one can check that $E$ is a projective localisation observable and $E^{\prime}$ is a normalised nonprojective one, both covariant with respect to $\pi$. Moreover, one has that $\sigma_{E}=\sigma_{E^{\prime}}$ is the two-dimensional trivial representation of the identity, although $E$ and $E^{\prime}$ are not equivalent.

By Eq. (32), one can always assume that localisation observables are normalised. The following corollary establishes a correspondence between normalised localisation observables $E$ and admissible maps $A$ satisfying Eq.(17) (compare with [17], [22], [31]). 
Corollary 3. Let $\pi$ be a representation of $G$, and let $\theta$ be as in Lemma 3. Given a normalised localisation observable $E$ covariant with respect to $\pi$, there is an admissible map A for $\pi$ with respect to $\left(H, \sigma_{E}\right)$ that satisfies $E q$.(7) and, for all $u, v \in \mathcal{D}_{\pi}$ and $Y \in \mathcal{B}(X)$,

$$
\langle E(Y) u, v\rangle=\int_{p^{-1}(Y)}\left\langle A \pi\left(g^{-1}\right) u, A \pi\left(g^{-1}\right) v\right\rangle \theta(g) d \mu_{G}(g) .
$$

In particular, $\pi$ is square-integrable modulo $\left(H, \sigma_{E}\right)$.

Conversely, if $\pi$ is square-integrable modulo $(H, \sigma)$ for some representation $\sigma$ of $H$ and $A$ is an admissible map for $\pi$ with respect to $(H, \sigma)$ such that Eq.(17) holds, then Eq.(33) defines a normalised localisation observable covariant with respect to $\pi$.

Proof. For the first part, due to Theorem 2, there is an isometry $W$ such that Eqs.(10) and (11) hold. In particular, $\pi$ is square-integrable. Since $W$ is an intertwining isometric operator, by Theorem 1 there is an admissible map $A$ such that Eq.(7) holds. By the definition of $E^{\sigma_{E}}$, Eq.(33) follows.

Conversely, assume that $\pi$ is square-integrable modulo $(H, \sigma)$ for some $\sigma$. By Corollary 1, there are admissible maps $A$ satisfying Eq.(7). In particular, the corresponding wavelet operator $W_{A}$ is isometric and intertwines $\pi$ and $L^{\sigma}$. Then, the map

$$
Y \mapsto W_{A}^{*} E^{\sigma}(Y) W_{A}=: E(Y)
$$

is a normalised localisation observable covariant with respect to $\pi$, explicitly given by Eq. (33).

The quoted result of Scutaru requires that $A$ is bounded and $A^{*} A$ is of trace class, whereas the one of Holevo is explicitly proven for $G$ compact with $H$ arbitrary and for $G$ a type I group with $H=\{e\}$.

\section{REFERENCES}

1. S. T. Ali, A general theorem on square-integrability: vector coherent states, J. Math. Phys. 39 (1999), 3954-3964. MR 99h:22015

2. S. T. Ali, J.-P. Antoine, and J.-P. Gazeau, Coherent States, Wavelets and Their Generalizations, Springer-Verlag, New York, 2000.

3. S. T. Ali, H. Führ, and A. E. Krasowska, Plancherel inversion as unified approach to wavelet transform and Wigner function, preprint math-ph/0106014.

4. R. J. Blattner, On induced representations, Amer. J. Math. 83 (1961), 79-98. MR 23:A2757

5. A. Borel, Représentations de groupes localement compacts, Lectures Notes in Mathematics No. 256, Springer-Verlag, New York, 1972. MR 54:2871

6. P. Busch, M. Grabowski, and P. Lahti, Operational Quantum Physics, Lecture Notes in Physics, New Series m: Monographs, vol. 31, 2nd corrected printing, Springer-Verlag, Berlin, 1997. MR 96j:81022 (1st ed.)

7. U. Cattaneo, On Mackey's imprimitivity theorem, Comment. Math. Helvetici 54 (1979), 629641. MR 81b:22009

8. D. P. L. Castrigiano and R. W. Henrichs, Systems of covariance and subrepresentations of induced representations, Lett. Math. Phys. 4 (1980), 169-175. MR 81j:22010

9. E. B. Davies, On the repeated measurement of continuous observables in quantum mechanics, J. Funct. Anal. 6 (1970), 318-346. MR 51:4896

10. J. Dieudonné, Elements d'analyse, Vol. 2, Gauthiers-Villars, Paris, 1968. MR 38:4247

11. J. Dieudonné, Elements d'analyse, Vol. 6, Gauthiers-Villars, Paris, 1975. MR 58:29825a

12. M. Duflo and C. C. Moore, On the regular representation of a nonunimodular locally compact group, J. Funct. Anal. 21 (1976), 209-243. MR 52:14145 
13. G. B. Folland, A Course in Abstract Harmonic Analysis, CRC Press, Boca Raton, 1995. MR 98c:43001

14. H. Führ and M. Mayer, Continuous wavelet transforms from semidirect products: Cyclic representations and Plancherel measure, J. Fourier Anal. Appl. 8 (2002), 375-397.

15. H. Führ, Admissible vectors for the regular representation, Proc. Amer. Math. Soc. 130 (2002), 2959-2970.

16. S. A. Gaal, Linear Analysis and Representation Theory, Springer-Verlag, Berlin, 1973. MR 56:5777

17. A. S. Holevo, On a generalization of canonical quantization, Math. USSR Izvestiya 28 (1986), 175-188. MR 87k:22006

18. A. S. Holevo, Probabilistic and Statistical Aspects of Quantum Theory, North Holland, Amsterdam, 1982. MR 85i:81038a

19. J. R. Klauder, Continuous representation theory I: postulates of continuous representation theory, J. Math. Phys. 4 (1963), 1055-1058. MR 27:2779

20. G. W. Mackey, Imprimitivity for representations of locally compact groups I, Proc. Natl. Acad. Sci. U.S.A. 35 (1949), 537-545. MR 11:158b

21. C. C. Moore, On the Frobenius reciprocity theorem for locally compact groups, Pacific J. Math. 12 (1962), 359-365. MR 25:5134

22. H. Moscovici and A. Verona, Coherent states and square integrable representations, Ann. Inst. Henri Poincaré 29 (1978), 139-156. MR 80d:22016

23. H. Neumann, Transformation properties of observables, Helv. Phys. Acta 45 (1972), 811-819. MR 53:7254

24. B. Orsted, Induced representations and a new proof of the imprimitivity theorem, J. Funct. Anal. 31, 355-359 (1979). MR 80d:22007

25. A. Perelomov, Generalized Coherent States and Their Applications, Springer-Verlag, Berlin, 1986. MR 87m:22035

26. N. K. S. Poulsen, Regularity Aspects of Infinite Dimensional Representations of Lie Groups, Ph. D. Thesis, M.I.T. Cambridge, Mass., 1970.

27. N. K. S. Poulsen, On $C^{\infty}$-vectors and intertwining bilinear forms for representations of Lie groups, J. Funct. Anal. 9 (1972), 87-120. MR 46:9239

28. B. Sz-Nagy, Extensions of Linear Transformations in Hilbert Space Which Extend Beyond this Space, Appendix to F. Riesz, B. Sz-Nagy, Functional Analyis, Frederick Ungar, New York, 1960. MR 16:837a MR 22:8338

29. D. J. Rowe, G. Rosensteel, and R. Gilmore, Vector coherent state representation theory, J. Math. Phys. 32 (1991), 2787-2791. MR 87a:81069

30. F. E. Schroeck, Quantum Mechanics on Phase Space, Kluwer Academic Publishers, Dordrecht, 1996. MR 97j:81004

31. H. Scutaru, Coherent states and induced representations, Lett. Math. Phys. 2 (1977), 101-107. MR 58:22406

32. W. F. Stinespring, Positive functions on $C^{*}$-algebras, Proc. Amer. Math. Soc. 6 (1955), 211216. MR 16:1033b

33. G. Warner, Harmonic Analysis on Semi-Simple Lie Groups I, Springer-Verlag, Berlin, 1972. MR 58:16979

Dipartimento di Fisica, Università di Genova, i.n.F.n., Sezione di Genova, Via DodeCANESo 33, 16146 Genova, Italy

E-mail address: cassinelli@genova.infn.it

Dipartimento di Matematica, Università di Modena, Via Campi 213/B, 41100 Modena, Italy and I.N.F.N., Sezione di Genova, Via Dodecaneso 33, 16146 Genova, Italy

E-mail address: devito@unimo.it 